\title{
Aprendizaje significativo en estudiantes que atienden el parto empleando un escenario virtual
}

\author{
Angie Nicol Cortes Moreno'; Oscar Eduardo Bedoya Osorio²
}

Asesora: María Neyfeth Posada Morales²

RESUMEN Este estudio trata de evidenciar el aprendizaje en estudiantes que atienden el parto, empleando el escenario virtual Areandina, el cual es un software producto de una investigación, que vienen utilizando los estudiantes de Enfermería como un medio de entrenamiento y fortalecimiento de sus competencias.

Método: estudio cuanti-cualtitativo descriptivo, con participación de 90 estudiantes de Enfermería Areandina, que respondieron una encuesta para evidenciar condiciones significativas del escenario virtual de atención del parto, después de su uso, basadas en la teoría de Ausubel.

Resultados: los estudiantes perciben en el escenario virtual de atención del parto, significado psicológico, contextual, social y clínico, que los motiva a aprender.

Conclusiones: los estudiantes inmersos en el escenario virtual de atención del parto virtual, comprenden mejor este fenómeno, ya que los contextualiza, motiva y permite poner en práctica sus conocimientos, antes de enfrentarse a situaciones reales.

Aprendizaje;

PALABRAS Parto Obstétrico;

CLAVE

Educación en Enfermería;

Programas Informativos (software).

1 Estudiantes Programa de Enfermería VIII semestre. Fundación Universitaria del Área Andina Seccional Pereira.

acortes30@estudiantes.areandina.edu.co, obedoya4@estudiantes.areandina.edu.co

2 Asesoramposada@areandina.edu.co 


\section{Significant learning in students who attend the birth by using a virtual scenario}

\begin{tabular}{l|l} 
ABSTRACT & $\begin{array}{l}\text { The objective of this study is to show significant learning in students who } \\
\text { attend childbirth using the Areandina virtual scenario, which is a software } \\
\text { product of previous research, which nursing students have been using as a } \\
\text { means of training and strengthening their skills. }\end{array}$
\end{tabular}

Methods: descriptive quantitative qualitative study, with the participation of 90 students of Areandina Nursing, who answered a survey to show significant conditions of the virtual stage of delivery care, after use, based on Ausubel's theory.

Results: students perceive in the virtual stage of childbirth care: psychological, contextual, social and clinical significance, which motivates them to learn.

Conclusions: students immersed in the virtual stage of virtual birth care, understand this phenomenon better, since it contextualizes, motivates and allows them to put their knowledge into practice, before facing real situations, which confirms that there is significant learning.

Learning;

Obstetric Delivery;

Nursing Education;

Information Programs (software). 


\section{INTRODUCCIÓN}

De acuerdo a la Resolución 3280 (1), la atención del parto de bajo riesgo en Colombia debe darse preferiblemente en un ámbito institucional, en donde se contará con un espacio físico dotado o equipado con todos los elementos necesarios para la atención del parto normal o la urgencia que pudiera presentarse, disponiendo también de talento humano como lo es el profesional de enfermería o médico, el cual debe estar altamente capacitado en la atención del primer periodo del parto, atención del expulsivo, atención del alumbramiento, atención del recién nacido y del puerperio inmediato. Lo que indica una gran responsabilidad por parte de la comunidad académica en la formación de los estudiantes en esta área de la salud.

No obstante, el aprendizaje relacionado con la atención del parto se ve afectado, porque la práctica real conlleva riesgos para los pacientes si son realizados por personal en formación, razón por la cual en las instituciones educativas donde se forman estudiantes de enfermería, se debe disponer de espacios físicos para la atención (Centros de Simulación Clínica), pero en Areandina esos espacios son insuficientes, debido a la gran demanda de estudiantes, además que es difícil mantener el interés y motivación por parte del aprendiz. Ante esta situación Areandina viene promoviendo el auto-entrenamiento en atención del parto de forma virtual, utilizando un software educativo que se elaboró con base a las necesidades identificadas dentro de la comunidad estudiantil y docente de la Institución, y que se basa en recomendaciones normativas vigentes, esperando que el estudiante ponga en práctica sus conocimientos.

Esta nueva forma de atención del parto virtual, introduce al estudiante en un entorno multisensorial, donde percibe la existencia de un espacio institucional en $3 \mathrm{D}$, dotado con todos los equipos y materiales necesarios para llevar a cabo las actividades propias del proceso. Para esto, el enfermero debe comprender la parte de su rol en la sala de partos, consistente en verificar la disponibilidad de estos equipos (incubadora, lámpara de calor radiante, fuentes de oxígeno, carro de paro adulto y neonatal entre otros), y para ello, utilizar una lista de chequeo que aparece en la pantalla, la cual si se encuentra incompleta no deja avanzar al estudiante en la atención del parto, ya que el objetivo es garantizar que ese espacio es seguro para ello.

Por otra parte, el escenario de parto virtual ubica al estudiante como parte del personal de salud que lo atiende, dándole responsabilidades que debe asumir en la práctica real, es decir, cumplir con las normas de bioseguridad, de asepsia y antisepsia, manejo de elementos estériles, y debe proceder en forma ordenada a atender el parto, realizando actividades propias de cada periodo del mismo, y que son fundamentales en la atención de calidad y seguridad del sujeto de cuidado. En cuanto a la parte humana del proceso, el escenario cuenta con un acompañante elegido por la mujer que se ubica en el lado derecho junto a la cabecera, al igual que no se observa intervencionismo o medicalización, y se respetan los derechos maternos, en el sentido que se permite que la mujer exprese sus sentimientos de dolor a través de la forma como puja o bien en la expresión de sus facies, que por cierto tienen fisonomía y rasgos latinos. 
Desde la evidencia, Riquelme y colaboradores (2), consideran que sin simulación clínica o virtual, no sería posible que el estudiante alcance hoy en día un buen grado de comprensión de los fenómenos que requieren aprenderse, ya que la neurociencia ha demostrado que estos escenarios por la gran cantidad de estímulos multisensoriales paralelos, activan la memoria y la metacognición, lo que conlleva a impulsar redes sinápticas generales o específicas, que en su proceso de interconexión contribuyen en el aprendizaje. Carbajo (3). Razones de peso que exaltan el escenario virtual de atención del parto como una oportunidad para que el estudiante se entrene independiente, aplique los conocimientos y adquiera habilidades.

Ahora bien, si se tiene en cuenta que la motivación es un elemento esencial e inherente para la marcha del aprendizaje, Jiménez (4), explica que cuando el estudiante accede a un objeto virtual de aprendizaje seguro, amigable y con contenido relevante, es decir, que observa sentido y significado al conocimiento, se estimula su disponibilidad para aprender. Por lo tanto, si los escenarios de atención del parto no tienen estas condiciones, se afectará negativamente el proceso de asimilación aprendizaje, máxime si no se dispone de tiempo y espacio para reforzar su aprendizaje en forma presencial, como tradicionalmente se hace en los centros de simulación clínica.

Respecto al aprendizaje significativo, Rodríguez (4), retoma los conceptos ausubelianos, considerando que los estudiantes no comienzan su aprendizaje desde cero, sino que lo van construyendo con sus vivencias y experiencias, lo cual manejado adecuadamente sirve para mejorar el proceso mismo de aprendizaje y hacerlo significativo. Sin embargo, la consecución de este tipo de aprendizaje supone el uso de materiales con un gran contenido psicológico y motivacional. En este sentido el material de aprendizaje debe activar los conocimientos previos para dar paso a los conocimientos nuevos. Logro que se espera alcancen los usuarios del escenario virtual de atención de parto, porque en el proceso se refuerzan los lineamientos establecidos en la normatividad vigente como la Guía de Práctica Clínica (6), La Resolución 3280 (7), entre otras, y la idea es que los ponga en práctica a medida que avance en el escenario virtual de atención al parto. Porque solo en ese momento comprenderán que sin conocimiento y sin práctica no podrán atender adecuadamente un parto en el contexto real, lo que puede despertar su interés y su motivación.

En conclusión, y como lo expresa Sarria (8), cada contenido teórico lleva implícita una habilidad o capacidad que el estudiante debe evidenciar para otorgarle un valor a su uso, y eso lo logra si se le ofrece la oportunidad de entrenamiento y acción; por consiguiente, los softwares educativos se han convertido en un referente para el desarrollo de habilidades intelectuales y procedimentales con un alto contenido motivacional Vázquez et al (9), y el software de atención del parto no es la excepción. Por eso surge la pregunta de investigación ¿hay aprendizaje significativo cuando los estudiantes de enfermería atienden el parto empleando el escenario virtual Areandina? 


\section{MÉTODO}

Tipo de estudio: la pregunta de investigación orienta el estudio para ser desarrollado bajo un enfoque de corte cuanti-cualitativo de naturaleza descriptiva, porque busca comprender las condiciones significativas del escenario virtual de atención del parto para relacionarlos con las competencias enfermeras, que es lo que le otorga significatividad a proceso.

Población y Muestra: la población objeto de estudio, fue conformada por 90 estudiantes de cuarto semestre, correspondientes a un grupo de la asignatura de Enfermería a la madre, pareja y recién nacido, del Programa de Enfermería de Areandina Pereira; seleccionado por conveniencia.

Instrumento: encuesta virtual con 15 preguntas cerradas y tres opciones de respuesta (totalmente de acuerdo, un poco de acuerdo y total desacuerdo) y espacio para justificación de su respuesta.

Técnica de Recolección de dato: los datos obtenidos virtualmente se llevaron a un drive y se analizaron las respuestas de acuerdo a categorías.

\section{RESULTADOS}

\section{Gráfico N. 1.}

Caracterización de la Población participante en el estudio.

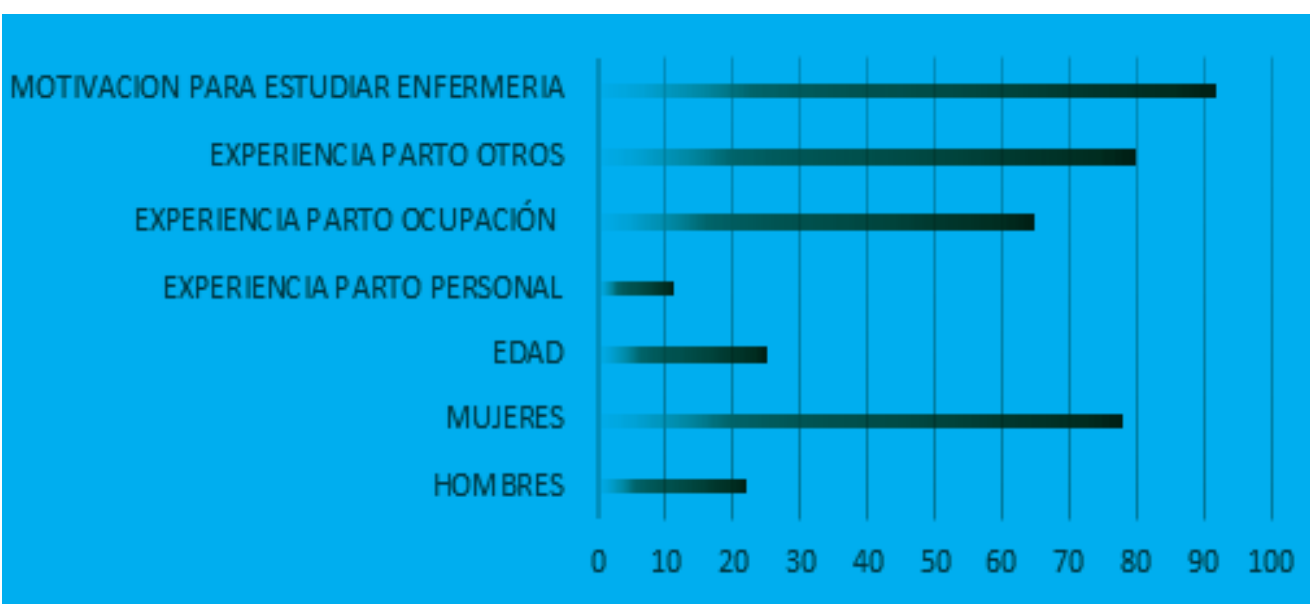

Fuente: Base de datos del estudio $(\mathrm{n}=90)$

La gráfica $\mathrm{N}^{\circ} 1$ evidencia que los estudiantes de enfermería en general tienen un factor motivacional importante, el cual es un elemento a considerar en el proceso de enseñanza aprendizaje, si se entiende que hay disposición e intención para aprender. Desde la evidencia científica, Alemán y Colaboradores (10), explican cómo 
habilidades y capacidades, en la formación del carácter y la moral en los estudiantes universitarios; lo que es constatado por Buitrago (11), pues alude que la principal motivación para estudiar Enfermería radica en la vocación de servicio y el propósito de cuidar, pero su mayor temor es enfrentar situaciones relacionadas con la atención directa a los pacientes, lo que explica la necesidad de un entrenamiento previo antes de las prácticas con pacientes, lo que se logra en los espacios de simulación clínica o virtual.

Por otra parte, el hecho de que los estudiantes tengan experiencias o vivencias propias o adquiridas a través de otras personas, como se observa en gráfica $\mathrm{N}^{\circ} 1$., es transcendental en la conexión de saberes previos con los saberes nuevos, porque estos, orientados adecuadamente, permiten dar significado a su rol dentro de la atención de la mujer durante el parto. Al respecto Mota y Valles (12), consideran que ambos saberes (previo y nuevo), le otorgan más sentido a lo que se aprende, se ubican en la memoria a largo plazo, de manera que pueden ser 'evocarlos' y utilizados cuando sea oportuno o se necesite su aplicación a un corto, mediano o largo plazo y en cualquier aspecto de su vida.

\section{Tabla $\mathbf{N}^{\circ} 1$.}

Condiciones de Aprendizaje significativo con la atención del parto empleando un escenario virtual.

\begin{tabular}{|c|c|c|c|c|}
\hline \multicolumn{5}{|c|}{ ESCENARIO VIRTUAL DE ATENCIÓN DEL PARTO "AREANDINA } \\
\hline \multirow{2}{*}{$\begin{array}{l}\text { Condiciones de la teoría } \\
\text { del aprendizaje } \\
\text { significativo de Ausubel }\end{array}$} & \multirow{2}{*}{$\begin{array}{c}\text { condiciones significativas } \\
\text { del escenario virtual de } \\
\text { atención del parto }\end{array}$} & $\begin{array}{l}\text { Totalmente } \\
\text { de acuerdo }\end{array}$ & $\begin{array}{l}\text { Algo de } \\
\text { acuerdo }\end{array}$ & $\begin{array}{c}\text { Totalmenteen } \\
\text { desacuerdo }\end{array}$ \\
\hline & & $\%$ & $\%$ & $\%$ \\
\hline \multirow{3}{*}{$\begin{array}{l}\text { Significatividad lógico y } \\
\text { psicologica del material }\end{array}$} & Significatividad contextual & 67 & 21 & 2 \\
\hline & Significatividad social & 76 & 10 & 4 \\
\hline & Significativadad Clínica & 81 & 0 & 9 \\
\hline
\end{tabular}

Fuente: Base de datos del estudio $(\mathrm{n}=90)$

La tabla No.1 evidencia que para los estudiantes que se sumergieron en el escenario virtual, este representa un "material" psicológicamente muy atractivo, porque los introduce en un espacio institucional de atención del parto, igual a los que realmente existen en el país, lo que les permite familiazarse fácilmente con el lugar, dándoles seguridad para actuar en él, y esto desde la óptica Ausubeliana denota "significatividad contextual", más aún cuando los estudiantes afirmaron que la sala les proporcionaba información visual y auditiva muy sugestiva, como equipos médicos, materiales y carteles que son representativos dentro de cualquier sala de partos. Igualmente afirmaron que se sintieron parte del escenario, al ser ellos mismos quienes atendieron el parto y tuvieron que aplicar las normas de bioseguridad, de asepsia y antisepsia, con 
las cuales ya están habituados y que, por ser significativas en la carrera profesional, las han interiorizado en la memoria.

Para los estudiantes también fue importante seguir los pasos en la atención del parto, ya que cada periodo significa reconocer unos signos en la parturienta, que indican la necesidad de establecer cuidados específicos, contemplados en los lineamientos de Minsalud, como son la protección del periné, la extracción de la placenta o su revisión. Igualmente, el establecer contacto piel a piel del recién nacido con la madre, pinzar le cordón umbilical, hacer el secado corporal céfalo-caudal, y otros.

La significancia social como factor actitudinal y ético del enfermero, la percibieron los estudiantes desde la responsabilidad que se asume con los dos sujetos de cuidado (madre e hijo), y en esto fue importante escuchar que la madre se "quejaba" y hacía el esfuerzo para pujar, y que aquí la mujer no representa estereotipos de belleza ya que conserva rasgos muy latinos, lo cual hizo que el escenario representara la realidad cotidiana.

Por último, la significancia clínica (81\%) les permitió darse cuenta que en la atención del parto no solo se requiere tener habilidades y destrezas cognitivas, habilidades procedimentales y actitudinales, sino también de gestión, de aplicación de la normatividad, lo cual expresaron utilizando términos como "el enfermero tiene que supervisar y hacer muchas cosas en la sala de partos", "aquí se siguen todos los pasos y normas de la guía del Ministerio" "yo ya sé atender un parto". Lo que significa comprensión del proceso, que, desde Ausubel, es aprendizaje significativo.

\section{DISCUSIÓN}

Rodríguez (13), confirma la necesidad de introducir nuevas metodologías en la enseñanza para facilitar la transmisión de la información, la asimilación y retención de los contenidos y lograr aprendizajes con mayores significados, incluso en el ámbito de las ciencias de la salud, pues reúne componentes especiales, es decir, que son disciplinas teórico-prácticas y el estudiante requiere ganar competencias en ambas, para poder fijar el aprendizaje.

Según Bolívar (14), todo material que se utilice en el proceso enseñanza, tiene que tener "significatividad psicológica" para que el estudiante comprenda lo que está "aprendiendo" y su utilidad en la vida profesional. Por lo tanto, debe ser capaz de generar pensamiento crítico (estimulación del análisis y reflexión), epistemología (consideración de los supuestos o filosofía de cada disciplina) y motivación; aspectos que contrastan con lo que los estudiantes percibieron en el escenario virtual de parto al apreciar significancia contextual, clínica y social.

Finalmente es transcendental anotar que el aprendizaje de los procederes inherentes a la profesión de enfermería o de medicina, pueden ser aprovechados cuando se usan softwares educativos como por ejemplo el escenario virtual de parto, ya que cuando son evaluados muestran grandes avances en el perfeccionamiento de habilidades 
teórico prácticas, superiores al $60 \%$ como lo confirma un estudio realizado por Arenas y colaboradores (15). Esto demuestra que, si se presentan contenidos en forma contextualizada, organizada y con aspectos muy relevantes como lo expresó la población objeto de este estudio, es más fácil comprender el proceso.

\section{CONCLUSIONES}

Este escenario virtual de atención del parto, demuestra que enfermería debe incursionar en la ciencia de la tecnología para motivar el estudiante y generar comprensión del proceso del parto, antes de llegar a su práctica real, porque los contextualiza, les permite percibir funciones, roles, aspectos normativos, técnicos, procedimentales y actitudinales entre otros, que le ayudan a dar significancia a lo aprendido desde el aula, es decir, a poner en práctica sus conocimientos.

1. Ministerio de Salud y Protección social. Gobierno de Colombia [Internet]. Resolución 3280 de 2 de agosto 2018. Bogotá [consultada diciembre 2017]. Disponible en:

https://www.minsalud.gov.co/Normatividad_Nuevo/Resoluci\%C3\%B3n\%20 No. $\% 203280 \% 20 \mathrm{de} \% 2020183280 . p d f$

2. Iqueleme, G; Acevedo, V; Muñoz, X. La metodología de la simulación en la enseñanza de los contenidos de parto y atención del recién nacido en enfermería. Revista Edu. Med Super [Internet]. 2017 [consultado agosto 2019]. Vol. 31(4):115. Disponible en:

http://scielo.sld.cu/scielo.php?script=sci_arttext\&pid $=$ S0864-21412017000400009

3. Carbajo, M. La sala de estimulación multisensorial Tabanque Revista Pedagógica [Internet]. 2014 [consultado abril 2019]. 27. Valladolid. Disponible en: https:// dialnet.unirioja.es/servlet/articulo?codigo $=5084331$

4. Jiménez, L. Reflexiones sobre motivación, aprendizaje y comunicación. Revista Virtual Universidad Católica del Norte [Internet]. 2011 [consultado febrero 2019]. 1(17). Disponible en:http://revistavirtual.ucn.edu.co/index.php/ RevistaUCN/article/view/220 
5. Rodríguez, M. La teoría del aprendizaje significativo: una revisión aplicable a la escuela actual. IN. Revista Electrònica d'Investigació i Innovación Educativa i Socioeducativa, [Internet]. 2011 [consultado febrero 2019]. 3(1). Disponible en: https://elibros.octaedro.com/appl/botiga/client/img/10112.pdf

6. Ministerio de Salud y Protección social. Colciencias. Gobierno de Colombia [Internet]. Guía de Práctica Clínica para la prevención, detección temprana y tratamiento de las complicaciones del embarazo, parto y puerperio. Guías 1115. 2013. Bogotá [consultada diciembre 2017]. Disponible en: http://gpc.minsalud.gov.co/gpc_sites/Repositorio/Conv_500/GPC_embarazo/ GPC_embarazo_completa.aspx

7. Op Cit. Resolución 3280/2018

8. Sarria E., J. Aprendizaje significativo y significatividad del aprendizaje. Acta Herediana. [Internet]. 2014 [consultado febrero 2019]. V.54. disponible en: http://www.upch.edu.pe/vrinve/dugic/revistas/index.php/AH/article/ viewFile/2275/2241

9. Vásquez, JA.; Rodríguez, M.; Marín, R. El software educativo en el proceso de enseñanza-aprendizaje de la disciplina Histórica de Cuba. EDUMECENTRO [Internet]. 2012 [consultado febrero 2019]. 4(3):64-72. Disponible en: http://scielo.sld.cu/pdf/edu/v5n1/edu05113.pdf

10. Alemán, B.; Navarro, O.; Suárez, R.; Izquierdo, B.; Encinas, T. La motivación en el contexto del proceso enseñanza aprendizaje en carreras de las ciencias de la salud. [Internet]. 2018 [consultado abril 2019]. 40(2). Matanzas. Disponible en: http://scielo.sld.cu/pdf/rme/v40n4/rme320418.pdf

11. Buitrago E., M.T. Razones para estudiar enfermería y dudas sobre la profesión. Las voces de los estudiantes. Investigación en Enfermería: Imagen y desarrollo [Internet]. 2008 [consultado febrero 2019]. 10(1):21-38. Disponible en: https://revistas.javeriana.edu.co/index.php/imagenydesarrollo/article/ view/1594

12. Mota, D.; Valles, R. Papel de los conocimientos previos en el aprendizaje de la matemática universitaria Acta Scientiarum. Education [Internet]. 2015 [consultado febrero 2019]. 37(1). Brasil. Disponible en: https://www.redalyc.org/articulo.oa?id=303332696010

13. Rodríguez, L. Metodologías de enseñanza para un aprendizaje significativo. Revista digital universitaria Unam.mx. [Internet]. 2014 [consultado febrero 2019]. 15(11). Disponible en: http://www.revista.unam.mx/vol.15/num11/ art90/art90.pdf

14. Bolívar, M.R. Temas para la educación. Revista digital para profesionales de la enseñanza 2009 [Internet]. №3 [consultado marzo 2019]. Disponible en: https://www.feandalucia.ccoo.es/docu/p5sd5097.pdf

15. Arenas, R.; García, K.; Díaz, M. El desarrollo de habilidades teórico prácticas en las carreras de medicina y enfermería con la utilización del software educativo. Revista Cubana de Tecnología de la Salud [Internet]. 2017. [consultado marzo 2019]. 8(4). Disponible en:

http://www.medigraphic.com/pdfs/revcubtecsal/cts-2017/cts174c.pdf 


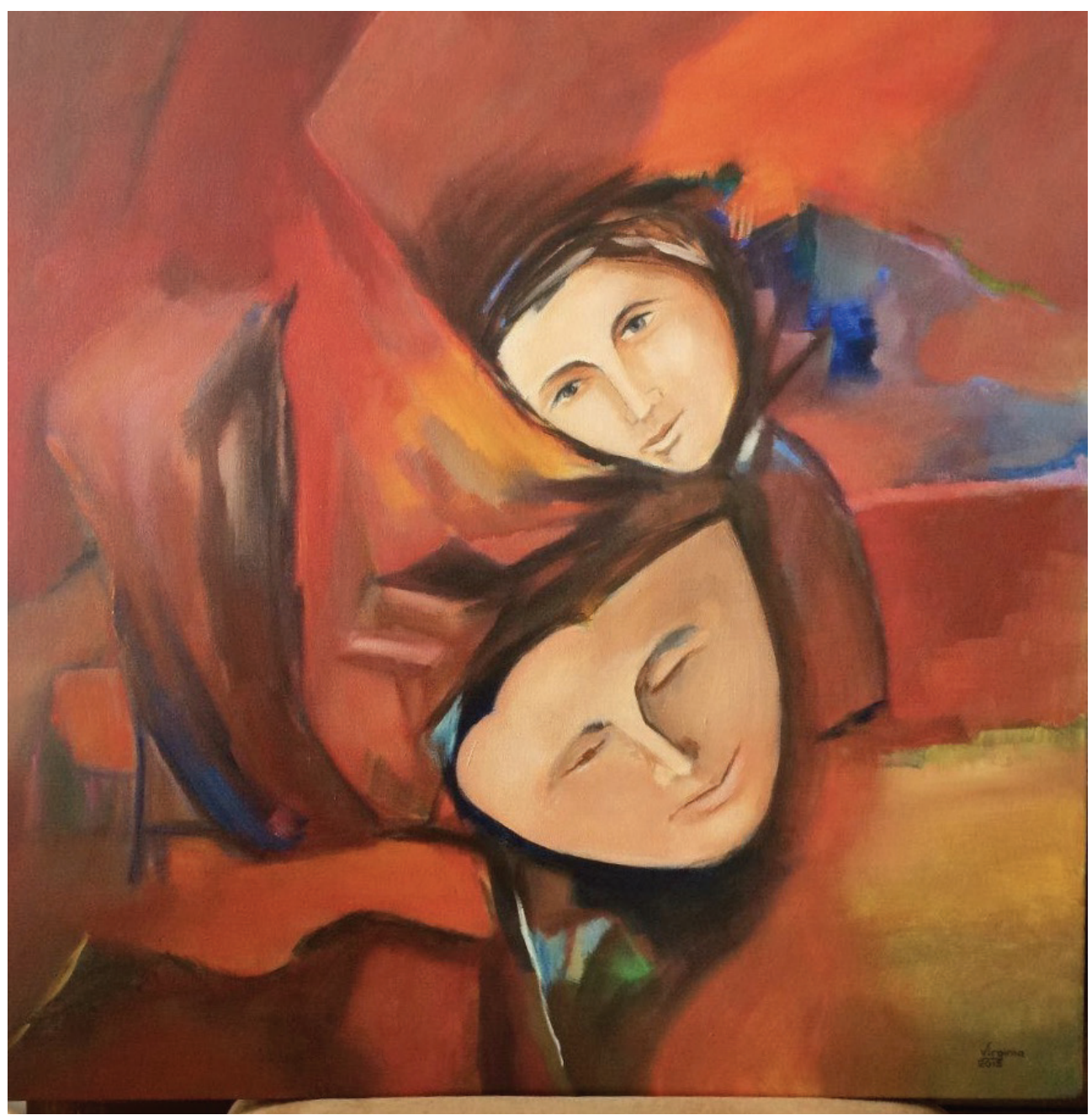

Acrílico sobre lienzo. “Comedia-tragedia”, Virginia Aristizábal Parra. 